\title{
Death receptor 5-mediated TNFR family signaling pathways modulate $\gamma$-humulene-induced apoptosis in human colorectal cancer HT29 cells
}

\author{
YU-HSUAN LAN $^{1}$, YANG-CHANG WU ${ }^{2}$, KAI-WEI WU ${ }^{1}$, JING-GUNG CHUNG ${ }^{3}$, \\ CHI-CHENG LU ${ }^{5}$, YUAN-LIANG CHEN ${ }^{6}$, TIAN-SHUNG WU ${ }^{1}$ and JAI-SING YANG ${ }^{4}$ \\ ${ }^{1}$ School of Pharmacy, ${ }^{2}$ Graduate Institute of Integrated Medicine, ${ }^{3}$ Biological Science and Technology, \\ ${ }^{4}$ Department of Pharmacology, School of Medicine, China Medical University, Taichung 404; \\ ${ }^{5}$ Department of Life Sciences, National Chung Hsing University, Taichung 402; ${ }^{6}$ Depatment of Medical Technology, \\ Yuan-Pei Institute of Science and Technology, Hsinchu 300, Taiwan, R.O.C.
}

Received August 16, 2010; Accepted October 22, 2010

DOI: $10.3892 /$ or.2010.1087

\begin{abstract}
A component from Emilia sonchifolia (L.) DC, $\gamma$ humulene, was investigated. Significantly decreased cell viability of human colorectal cancer HT29 cells in a dosedependent manner with $\mathrm{IC}_{50} 53.67 \pm 2.99 \mu \mathrm{M}$ for $24-\mathrm{h}$ treatment was found. $\gamma$-Humulene induced apoptotic cell death and apoptosis was confirmed by morphological assessment. The staining with propidium iodide (PI) and flow cytometric analysis also showed that $\gamma$-humulene significantly promoted the sub-G1 phase (an apoptotic population) in HT29 cells. Colorimetric assays indicated that pretreatment with a specific inhibitor of caspase-8 (Z-IETDFMK) significantly reduced activities of caspase- 8 and caspase- 3 in examined HT29 cells. $\gamma$-Humulene stimulated the death receptor 5 (DR5), DR4, Fas-associated protein with death domain (FADD), the cleavage of caspase- 8 and cleavage caspase-3, but reduced the protein levels of cellular Fasassociated death-domain-like IL-1ß-converting enzyme inhibitory protein (c-FLIP) by Western blot analysis. Consequently, $\gamma$-humulene-triggered cell death was significantly attenuated by DR 5 siRNA and the caspase- 8 inhibitor. Based on our results, we suggest that $\gamma$-humulene induced apoptotic cell death in HT29 cells through a DR5mediated caspase-8 and -3-dependent signaling pathway.
\end{abstract}

Correspondence to: Dr Jai-Sing Yang, Department of Pharmacology, China Medical University, No 91, Hsueh-Shih Road, Taichung 40402, Taiwan. R.O.C.

E-mail: jaising@mail.cmu.edu.tw

Dr Tian-Shung Wu, School of Pharmacy, China Medical University, No 91, Hsueh-Shih Road, Taichung 40402, Taiwan, R.O.C.

E-mail: tswu@mail.cmu.edu.tw

Key words: $\gamma$-humulene, herbal medicine, TNFR family apoptotic signaling, death receptor 5, human colorectal cancer HT29 cells
Therefore, this agent might be useful for developing new therapeutic regimens for treatment of colorectal cancer in the future.

\section{Introduction}

Colorectal cancer is a major cause of deaths in the world, and it is the third leading cause of cancer death in Taiwan. About 19.6 per 100,000 people die per year of colorectal cancer, according to the reports of the Department of Health, R.O.C. (Taiwan) in 2009 (http://www.doh.gov.tw/EN2006/ index_EN.aspx). Fluorouracil (5-FU), oxaliplatin (FOLFOX), and irinotecan (CPT-11) are applied as chemotherapeutic agents in first-line therapy for colorectal cancer, but the side effects limit its clinical usefulness (1-3). Therefore, discovery of a new agent for anti-colorectal cancer from novel compounds dietary natural products and traditional Chinese medicine (TCM) provide a useful application and chemotherapeutic effectiveness on colorectal cancer $(4,5)$.

Two main pathways are involved in the apoptosis process, either the intrinsic mitochondria-mediated pathway or the extrinsic death receptor pathway (3). The tumor necrosis factor-related apoptosis-inducing ligand (TRAIL) binds to its receptors such as death receptor 4 (DR4; TRAIL-R1) and DR5 (TRAIL-R2) to activate the extrinsic death receptor pathway $(6,7)$. Interaction of TRAIL with DR4 and DR5 leads to formation of homo/hetero-complexes for recruitment and then activation of Fas-associated death domain (FADD), and activation of initiator caspases- 8 and effector caspase- 3 that is finally responsible for apoptosis $(8,9)$. The extrinsic apoptotic pathway is negatively regulated mainly by the cellular FLICE-inhibitory protein (c-FLIP). c-FLIP recruited to FADD in death-inducing signaling complex then prevents the activation of procaspase-8 (10).

Emilia sonchifolia (L.) DC (Compositae) is a folklore medicinal plant in India and China (11). It is also used against inflammation, rheumatism, cough, cuts and wounds (11-13). In a previous study, the methanolic extract of Emilia sonchifolia (L.) DC (Compositae) inhibited Dalton's 
lymphoma (DL), Ehrlich ascites carcinoma (EAC) and mouse lung fibroblast (L-929) cells. However, it is not toxic to normal human lymphocytes in vitro. In vivo study also showed that oral administration of the extract (100 mg/kg, body weight) to mice reduced the development of both solid tumors and increased the life span of tumor-bearing mice (11). Furthermore, our preliminary result demonstrated that $\gamma$-humulene (Fig. 1A) is one of the components in Emilia sonchifolia (L.) DC (Compositae) by GC/Mass analysis. However, there is no report addressing the possible anti-colorectal cancer effects of $\gamma$-humulene. In this study, we investigated the mechanism of apoptosis induction by $\gamma$-humulene in HT29 cells. Based on our results, we suggest that $\gamma$-humulene induces apoptosis in human colorectal cancer HT29 cells via a DR5-mediated apoptotic pathway.

\section{Materials and methods}

Chemicals and reagents. $\gamma$-Humulene, MTT (3-[4,5dimethylthiazol-2-yl]-2,5-diphenyltetrazolium bromide), propidium iodide (PI), Tris-HC1 and Triton X-100 and RNase A were purchased from Sigma Chemicals Co. (St. Louis, MO, USA). Fetal bovine serum (FBS), L-glutamine, Lipofectamine 2000, penicillin-streptomycin, RPMI-1640 medium and trypsin-EDTA were obtained from Invitrogen Corp. (Carlsbad, CA, USA). Caspase-8 inhibitor (Z-IETDFMK) (R\&D systems, Minneapolis, MN, USA) was dissolved in DMSO and diluted in cell culture medium before use. Antibodies against caspase- 8 and caspase- 3 were purchased from Cell Signaling Technology (Beverly, MA, USA). Antibodies to Fas, FasL, FADD, cFLIP, GAPDH and DR5 siRNA were purchased from Santa Cruz Biotechnology Inc. (Santa Cruz, CA, USA). Antibodies against DR4 and DR5 were purchased from Novus Biologicals Inc. (Littleton, CO, USA).

Cell culture. Human colorectal cancer HT29 cell line was purchased from the Food Industry Research and Development Institute (Hsinchu, Taiwan). Cells were plated onto $75-\mathrm{cm}^{2}$ tissue culture flasks in RPMI-1640 medium supplemented with $10 \%$ FBS, 100 Units/ml penicillin, $100 \mu \mathrm{g} / \mathrm{ml}$ streptomycin and $2 \mathrm{mM} \mathrm{L}$-glutamine and grown at $37^{\circ} \mathrm{C}$ under a humidified $5 \% \mathrm{CO}_{2}$ and $95 \%$ air at one atmosphere (14).

Cell morphological examination. About $2 \times 10^{5}$ cells/well of HT29 cells onto 12-well plate were treated with 0, 25, 50, 75 and $100 \mu \mathrm{M}$ of $\gamma$-humulene and then were incubated for $24 \mathrm{~h}$. Cells were directly examined and photographed under a contrast-phase microscope (14).

Determination of cell viability by MTT assay. Approximately $2 \times 10^{4}$ cells/well of HT29 cells onto 96-well plates were exposed to $0,25,50,75$ and $100 \mu \mathrm{M}$ of $\gamma$-humulene, and DMSO, $0.1 \%$ in media served as a vehicle control. After a 24-h incubation, $100 \mu \mathrm{l}$ of MTT $(5 \mu \mathrm{g} / \mathrm{ml})$ solution was added to each well, and the plate was incubated at $37^{\circ} \mathrm{C}$ for $4 \mathrm{~h}$. One hundred microliters of $0.04 \mathrm{~N} \mathrm{HCl}$ in isopropanol was added and the absorbance at $570 \mathrm{~nm}$ was measured for each well. The cell survival ratio was expressed as $\%$ of control. All results are from three independent experiments (15).
Apoptotic cells analysis by flow cytometry. HT29 cells were seeded onto 12 -well cell culture plates at $2 \times 10^{5}$ cells/well and then incubated with $50 \mu \mathrm{M}$ of $\gamma$-humulene for $24 \mathrm{~h}$. The cells were harvested and washed by centrifugation. For apoptosis determination, cells were fixed by $70 \%$ ethanol in $-20^{\circ} \mathrm{C}$ overnight and then re-suspended in PBS containing $40 \mu \mathrm{g} / \mathrm{ml}$ PI, $100 \mu \mathrm{g} / \mathrm{ml}$ RNase A and $0.1 \%$ Triton X-100 in dark room for $30 \mathrm{~min}$. The apoptotic nuclei were determined by flow cytometry (FACSCalibur ${ }^{\mathrm{TM}}$, Becton-Dickinson, Franklin Lakes, NJ, USA). All results were performed from three independent experiments (16).

Assays for caspase-8 and -3 activities. Approximately $1 \times 10^{6}$ cells of HT29 cells onto 75-T flask were pretreated with or without the selective Z-IETD-FMK (a caspase- 8 inhibitor) and then were treated with $50 \mu \mathrm{M}$ of $\gamma$-humulene then incubated for $24 \mathrm{~h}$ to determine activities of caspase- 8 and -3 which were assessed according to the manufacturer's instructions (Caspase colorimetric kit, R\&D System Inc., Minneapolis, MN, USA). Briefly, cells were harvested and lysed in $50 \mu 1$ lysis buffer containing $2 \mathrm{mM}$ DTT for $10 \mathrm{~min}$. After centrifugation, the supernatants containing $100 \mu \mathrm{g}$ proteins were incubated with caspase- 8 and caspase- 3 substrate in reaction buffer. Samples were incubated in a 96-well flat bottom microplate at $37^{\circ} \mathrm{C}$ for $1 \mathrm{~h}$. Levels of released pNA (Z-DEVE-pNA and Z-IETD-pNA for caspase-3 and -8, respectively) were measured with an ELISA reader (Anthos Labtec Instruments, Salzburg, Austria) at OD405 nm. All results are from three independent experiments (17).

RNA interference transfection. HT29 cells were grown to $70 \%$ confluence in 6-well culture dishes, and LO GC Duplex Stealth RNAi Negative Control (100 nM) or DR5 siRNA (100 nM) was transfected using Lipofectamine 2000 according to the manufacturer's instructions (Invitrogen, Carlsbad, CA, USA). After transfection, cells were seeded, exposed to $50 \mu \mathrm{M}$ of $\gamma$-humulene for $24 \mathrm{~h}$ and then analyzed using Western blot analysis and MTT assay $(18,19)$.

Western blot analysis. HT29 cells $\left(2.5 \times 10^{7}\right)$ in 75 -T flask, were treated with $50 \mu \mathrm{M}$ of $\gamma$-humulene for $0,3,6$ and $12 \mathrm{~h}$. The cells were harvested and washed with cold 1X PBS. The total proteins were collected and measured as previously described (19). The protein concentration was measured by using a BCA assay kit (Pierce Biotechnology Inc., Rockford, IL, USA). Equal amounts of cell lysate were run on 10-12\% SDS-polyacrylamide gel electrophoresis (SDS- PAGE) and electrotransferred to a nitrocellulose membrane by using iBot $^{\mathrm{TM}}$ Dry Blotting System (Invitrogen). The blot was soaked in blocking buffer (5\% non-fat dry milk/0.05\% Tween-20 in $20 \mathrm{mM}$ TBS at pH 7.6) at room temperature for $1 \mathrm{~h}$ and then were incubated with anti-Fas, FasL, DR4, DR5, FADD, cFLIP, caspase- 8 , caspase-3 and GAPDH antibodies in blocking buffer at $4^{\circ} \mathrm{C}$ overnight. Membranes were washed three times with Tris-buffered saline/Tween-20 for $10 \mathrm{~min}$ and incubated with secondary horseradish peroxidase (HRP)conjugated antibody. The blots were developed using a chemiluminescence (ECL) detection kit (Millipore, Billerica, MA, USA) followed by development on Kodak Bio-MAX MR film (Eastman Kodak, Rochester, NY, USA). All results 
A

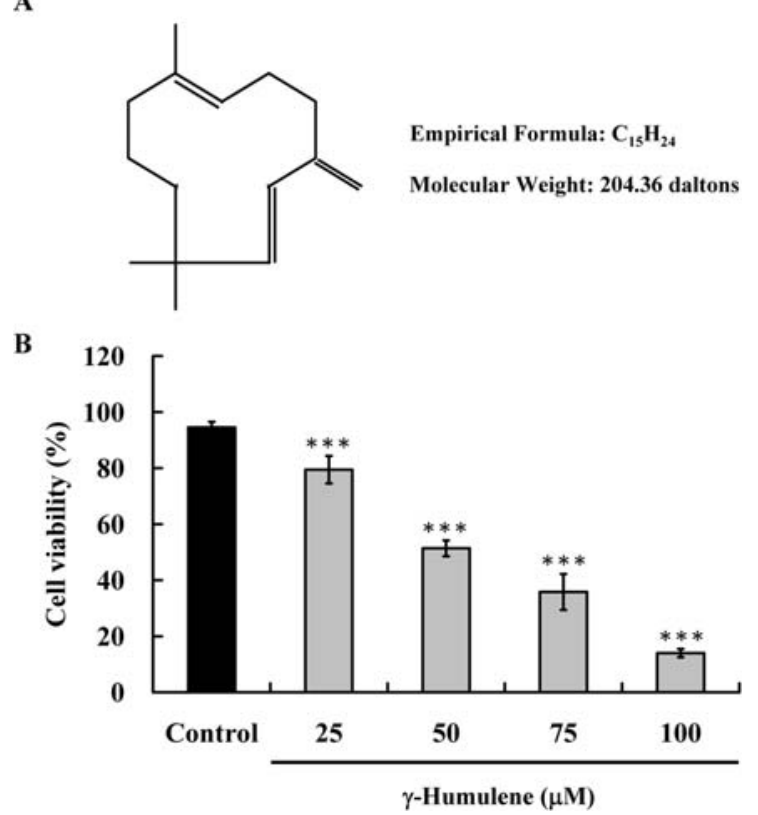

Figure 1. Effects of $\gamma$-humulene on the percentage of HT29 cell viability. The $\gamma$-humulene chemical structure, empirical formula and molecular weight (A). For cell viability assay, HT29 cells were cultured and treated with $0,25,50,75$ and $100 \mu \mathrm{M}$ of $\gamma$-humulene for $24 \mathrm{~h}$ and the percentages of viable cells were detected by MTT assay described in Materials and methods (B). There was a significant difference $\left({ }^{* * *} \mathrm{p}<0.001\right)$ when compared with the control group by Student's t-test analysis.

are from three independent experiments $(19,20)$. The relative image abundance of each band was quantified using $\mathrm{NIH}$ Image/ImageJ (version 1.43) for Windows (21).

Statistical analysis. Student's t-test was used to analyze the differences between the $\gamma$-humulene-treated and control groups. The data were expressed as mean $\pm \mathrm{SD}$ from at least three independent experiments. ${ }^{* * *} \mathrm{p}<0.001$ was indicative of significant difference.

\section{Results}

$\gamma$-Humulene reduces cell viability and induces growth inhibition in HT29 cells. We investigated the growth inhibition effect of $\gamma$-humulene on HT29 cells. In order to determine these effects on cell viability, HT29 cells were treated with different concentrations $(0,25,50,75$ and $100 \mu \mathrm{M})$ of $\gamma$-humulene, and cell number was counted daily. The results are presented in Fig. 1B and revealed that $\gamma$-humulene decreased cell viability of HT29 cells in a dose-dependent manner. The $\mathrm{IC}_{50}$ of $\gamma$-humulene was $53.67 \pm 2.99 \mu \mathrm{M}$ after 24-h treatment.

$\gamma$-Humulene triggers apoptotic cell death in HT29 cells. $\gamma$ Humulene-reduced cell viability due to cell apoptosis. Fig. 2A shows apoptotic morphological changes, including cell rounding and shrinkage after 24-h incubation with 25, 50, 75 and $100 \mu \mathrm{M}$ of $\gamma$-humulene. HT29 cells were cultured for $24 \mathrm{~h}$ in the absence or presence of $\gamma$-humulene $(25,50,75$ and $100 \mu \mathrm{M}$ ) and sub-G1 group (apoptotic population), which were analyzed by flow cytometry. The result is
A
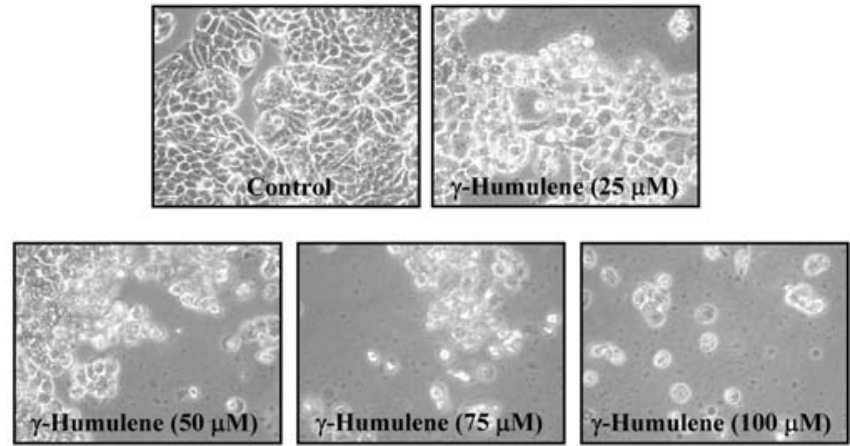

B

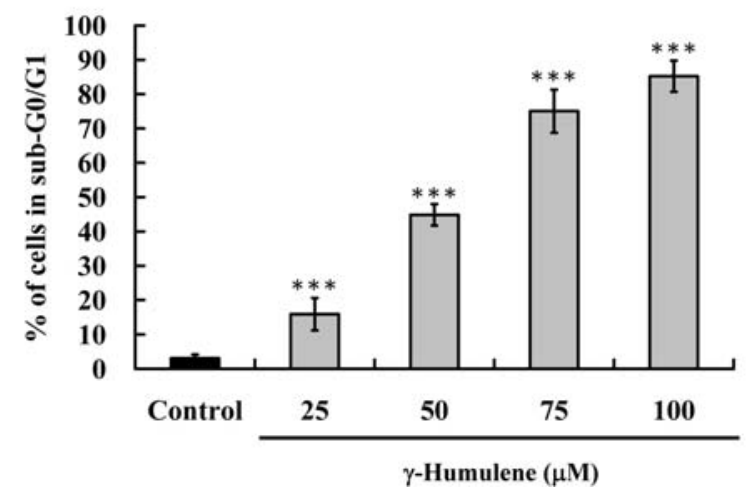

Figure 2. $\gamma$-Humulene effects on morphological changes and apoptosis. HT29 cells were cultured and exposed to $0,25,50,75$ and $100 \mu \mathrm{M}$ of $\gamma$-humulene for $24 \mathrm{~h}$. The cell morphological changes were examined and photographed under phase-contrast microscopy (x200) as described in Materials and methods (A). HT29 cells were cultured with 0, 25, 50,75 and $100 \mu \mathrm{M}$ of $\gamma$-humulene for $24 \mathrm{~h}$ and apoptosis was detected by flow cytometric analysis of PI staining. THe percent apoptosis measured in sub-G1 population $(B)$. There was a significant difference $\left({ }^{* * *} \mathrm{p}<0.001\right)$ when compared with the control group by Student's t-test analysis.

presented in Fig. 2B, indicating that $\gamma$-humulene increased $\%$ of cells in sub-G1 group (apoptosis) of HT29 cells in a dosedependent manner.

$\gamma$-Humulene activates caspase-8 and caspase-3 in HT29 cells. To investigate the mechanism of $\gamma$-humulene-induced apoptosis, we investigated the activities of caspase- 8 and -3 , respectively, in $\gamma$-humulene-treated HT2 29 cells. As shown in Fig. 3, $\gamma$-humulene caused an increase of caspase-8 (Fig. 3A) and caspase-3 (Fig. 3B) activity after 24-h treatment. However, the activity of caspase- 9 was not significant (data not shown). $\gamma$-Humulene-induced caspase- 8 and caspase- 3 activations were also significantly attenuated by a caspase- 8 specific inhibitor Z-IETD-FMK (Fig. 3A and B). Pre-incubation with Z-IETD-FMK significantly protected $\gamma$-humuleneinduced cell death and growth inhibition (Fig. 3C). Based on our results, we suggest that the caspase- 8 and caspase- 3 activation might be involved in $\gamma$-humulene-induced apoptotic cell death in HT29 cells.

$\gamma$-Humulene stimulates the levels of DR5 in HT29 cells. To determine the death receptor apoptotic signaling pathway of the $\gamma$-humulene-induced activation of caspase- 8 , the protein levels of Fas, FasL, DR4 and DR5 were determined by using 
A
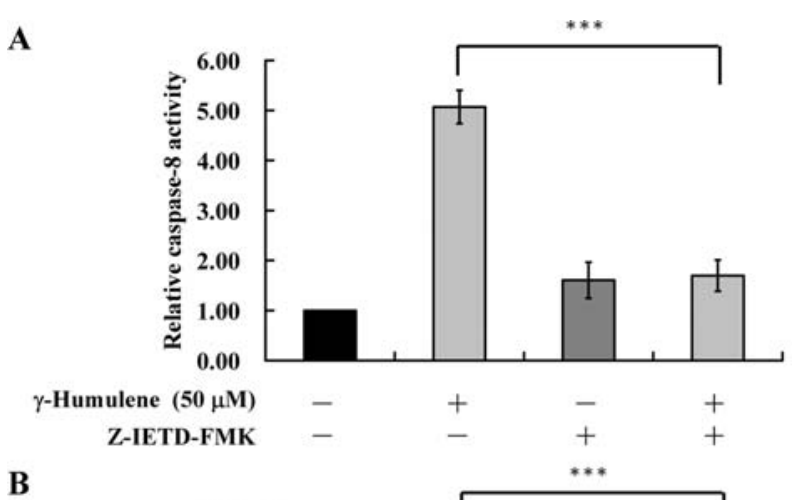

B

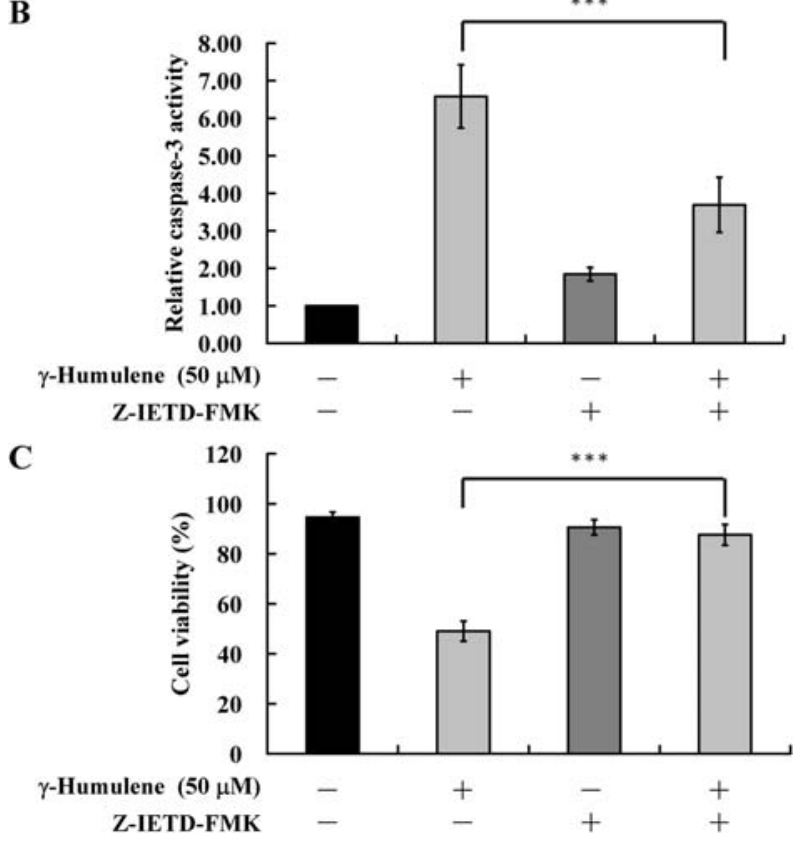

Figure 3. $\gamma$-Humulene promoted caspase-8 and caspase-3 activity. HT29 cells were pre-incubated with or without an inhibitor of caspase-8 (Z-IETD-FMK) for $3 \mathrm{~h}$ and then treated with $50 \mu \mathrm{M}$ of $\gamma$-humulene for $24 \mathrm{~h}$. $\gamma$-Humulene stimulated the activities of caspase- 8 (A), caspase- 9 (B) and the percentage of viable cells were detected by MTT assay (C) in HT29 cells determined as described in Materials and methods. ${ }^{* * *} \mathrm{p}<0.001$, a significant difference by Student's t-test analysis.

Western blot analysis. As shown in Fig. 4A, the levels of DR4 and DR5 rather than Fas and FasL were significantly increased after $\gamma$-humulene treatment. Importantly, $\gamma$-humulene promoted the protein level of DR5 after exposure to $\gamma$-humulene for $3 \mathrm{~h}$, and the levels contributed to apoptosis in HT29 cells. To further evaluate whether or not DR5 has a major role in $\gamma$-humulene-induced apoptotic cell death in HT29 cells, we then used DR5 siRNA to block $\gamma$-humulene-induced DR5mediated cell death. As seen in Fig. 4B, DR5 siRNA is able to protect $\gamma$-humulene-tiggered cell death in HT29 cells. Thus, these results suggest that $\gamma$-humulene induced apoptosis in HT29 cells through DR5-regulated death receptor signaling pathways.

$\gamma$-Humulene alters signals associated with extrinsic death receptor apoptotic proteins. To study the mechanisms of $\gamma$-humulene-induced apoptotic cell death through deathreceptor signaling pathway, we investigated the protein expression levels of FADD, cFLIP, caspase- 8 and caspase- 3 proteins by Western blot analysis. As shown in Fig. 5,

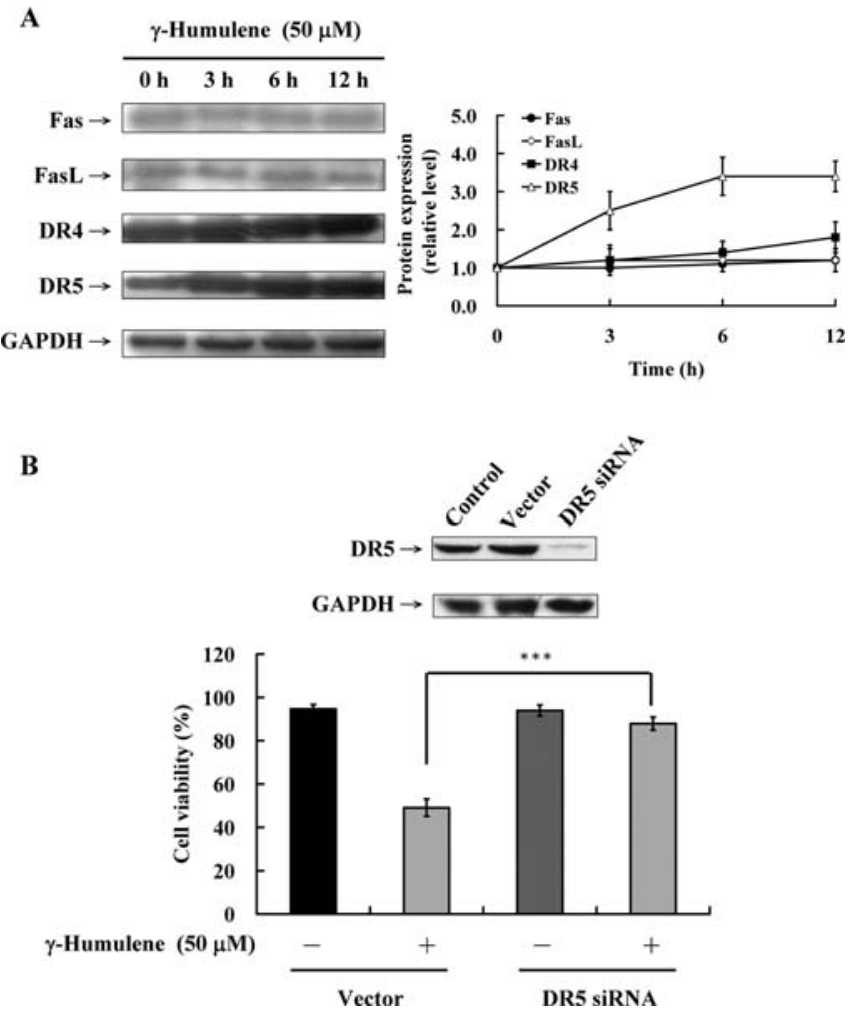

Figure 4. $\gamma$-Humulene-induced apoptotic cell death by up-regulation of DR5. HT29 cells were cultured with $50 \mu \mathrm{M}$ of $\gamma$-humulene for $0,3,6$ and $12 \mathrm{~h}$ and then harvested. Total proteins extracts were prepared, followed using primary antibodies against Fas, FasL, DR4 and DR5 (A). All proteins were detected by Western blotting (left panel) and protein levels were quantified by image analysis (right panel). Knockdown of DR5 by DR5 siRNA attenuated $\gamma$-humulene-induced cell death and growth inhibition. Top panel, total cellular lysates were subjected to Western blot analysis of DR5. Bottom panel, HT29 cells were treated with or without $50 \mu \mathrm{M}$ of $\gamma$-humulene in control siRNA or DR5 siRNA for $24 \mathrm{~h}$, and the cell viability was analyzed by MTT assay. ${ }^{* * *} \mathrm{p}<0.001$, a significant difference by Student's t-test analysis.

$\gamma$-humulene increased the levels of FADD, the cleavage of caspase- 8 and caspase- 3 and decreased the levels of c-FLIP. Our results suggest that $\gamma$-humulene-induced apoptosis is through a death receptor-mediated signaling pathway.

\section{Discussion}

The death receptor extrinsic apoptotic pathway has been proposed as a therapeutic target in human colorectal cancer $(22,23)$. New compounds, dietary natural products or traditional Chinese medicine (TCM) that could activate death receptor extrinsic apoptotic pathway should have therapeutic potential in human colorectal cancer treatment $(24,25)$. $\gamma$-Humulene is one of the common components in Emilia sonchifolia (L.) DC (Compositae) by GC/Mass analysis in our preliminary study. In this study, we first demonstrated that $\gamma$-humulene reduced cell growth in HT29 cells through induction of cell apoptosis. We also provided strong evidence that $\gamma$-humulene induced apoptosis in HT29 cells through the death receptor extrinsic apoptotic pathway. We showed in the present study that $\gamma$-humulene: i) decreased the percentage of viable cells; ii) induced apoptotic morphological changes; iii) up-regulated the protein levels of FADD, 


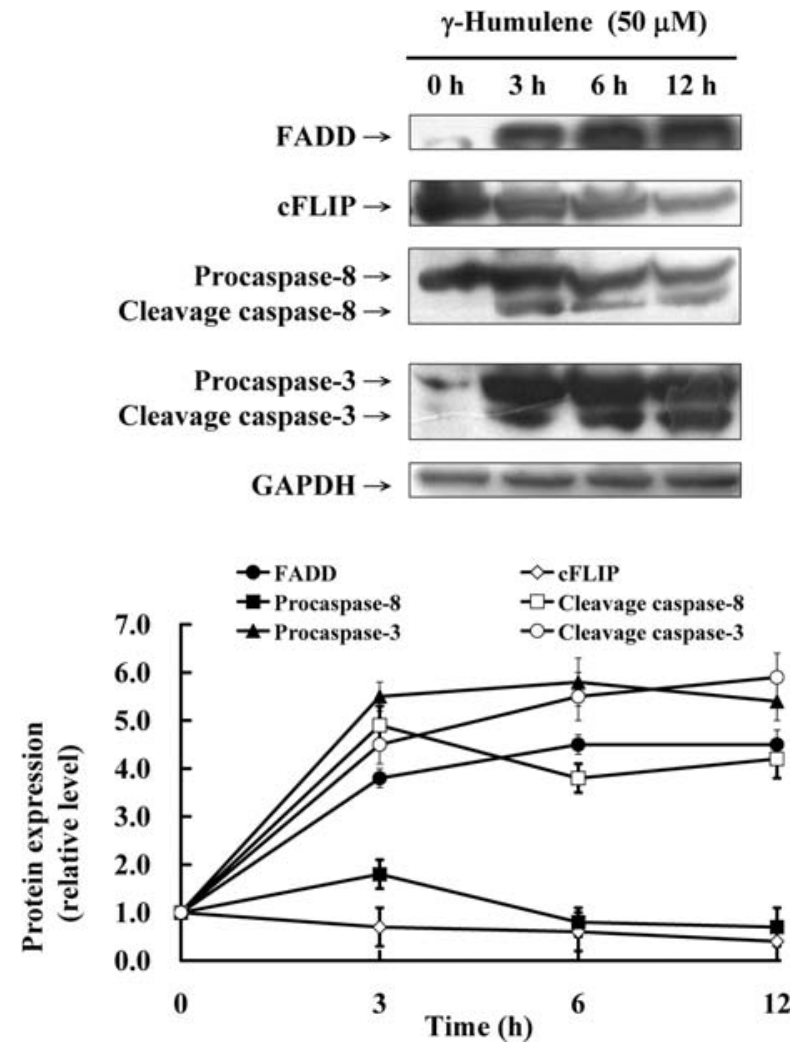

Figure 5. $\gamma$-Humulene regulates the death-receptor downstream apoptotic signaling molecule. HT29 cells were exposed to $50 \mu \mathrm{M}$ of $\gamma$-humulene for 0 , 3,6 and $12 \mathrm{~h}$ and then harvested. Total proteins extracts were prepared, by using antibodies against FADD, cFILP, caspase- 8 and caspase-3 (A). All proteins were detected by Western blot analysis (top panel) and protein levels were quantified by image analysis (bottom panel).

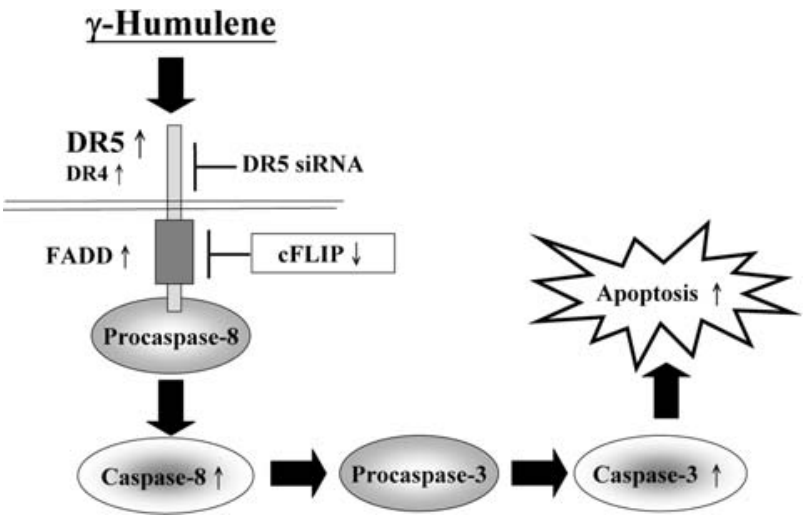

Figure 6. The proposed model of $\gamma$-humulene-induced death receptor 5 (DR5)-mediated apoptotic cell death in human colorectal cancer HT29 cells.

caspase- 8 and caspase- 9 and activates caspase- 8 , and -3 through DR5-mediated death receptor apoptotic pathway.

The transcriptional regulation of DR5 is complex and the multiple transcription factor binding sites of CHOP (CCAAT/ enhancer-binding protein-homologous protein), p53 and nuclear factor kappa-light-chain-enhancer of activated B cells $(\mathrm{NF}-\kappa \mathrm{B})$, are present in the DR5 upstream region $(26,27)$. Recently, it was also reported that p53 is a key regulator activating the extrinsic pathway through the induction of genes encoding transmembrane proteins such as DR4 and
DR5 (18). In this study, our results demonstrated that the $\gamma$ humulene significantly increased the protein level of DR5 (Fig. 4A) in HT29 cells. In addition, knockdown of DR5 expression by DR5 siRNA significantly inhibited the cell growth-inhibitory effects after treatment with $\gamma$-humulene in HT29 cells (Fig. 4B). According to our results, we suggest that p53 might be involved in $\gamma$-humulene up-regulated DR5mediated extrinsic apoptotic signaling pathways.

Cysteine-aspartic proteases (caspases) play an important role in apoptosis. Two major signaling pathways are activated caspase- 8 and increased apoptotic signals: i) directly cleaving and activating downstream caspase-3 or caspase-7; ii) stimulating the intrinsic mitochondrial pathway by processing Bid (28). Bid is a member of the Bcl-2 family bridging the apoptotic interaction between the membrane death receptors and the mitochondria. The truncated tBid protein can then disrupt mitochondrial membrane potential leading to the release of cytochrome c, Apaf-1, pro-caspase-9, AIF and Endo $\mathrm{G}$ into the cytosol activating the caspase-9 and-3 (29). Our results demonstrated the $\gamma$-humulene significantly promoted activities of caspase- 8 (Fig. 3A) and caspase- 3 (Fig. 3B) in 24-h treatment, but there was no significant effect on caspase-9 activity (data not shown) in HT29 cells. In addition, $\gamma$-humulene induced activations of caspase- 8 and caspase- 3 in examined cells and the growth inhibition was significantly attenuated by a specific caspase- 8 inhibitor (Fig. $3 \mathrm{~A}$ and $\mathrm{B})$. Our results suggest that DR5 death receptormediated pathway is the major pathway of $\gamma$-humuleneinduced apoptosis in HT29 cells.

The proposed model of $\gamma$-humulene-induced death receptor 5-mediated TNFR family apoptotic pathways in human colorectal cancer HT29 cells is shown in Fig. 6. In conclusion, our results suggest that $\gamma$-humulene has anticancer activity by stimulating the clustering of DR4/DR5 and associated FADD protein levels, lead to caspase- 8 and caspase- 3 activation, and then induction of apoptosis in HT29 cells. Therefore, our study is likely to provide the basis for further anti-colorectal cancer development of $\gamma$-humulene.

\section{Acknowledgements}

The investigation was supported by a research grant from the National Science Council of the Republic of China (95-2320B-039-049-MY2; NSC 97-2320-B-039-020-MY3) and a grant from China Medical University, Taichung, Taiwan (CMU97184).

\section{References}

1. Ocvirk J, Brodowicz T, Wrba F, et al: Cetuximab plus FOLFOX6 or FOLFIRI in metastatic colorectal cancer: CECOG trial. World J Gastroenterol 16: 3133-3143, 2010.

2. Laszlo L and Tamas N: The development of the first line treatment of metastatic colorectal cancer (mCRC). Magy Onkol 53: 237-246, 2009.

3. Gravalos C, Garcia-Escobar I, Garcia-Alfonso P, Cassinello J, Malon D and Carrato A: Adjuvant chemotherapy for stages II, III and IV of colon cancer. Clin Transl Oncol 11: 526-533, 2009.

4. Rocken C: Molecular targets for colon cancer. VEGF, EGFR, and what else? Pathologe 29 (Suppl. 2): 200-203, 2008.

5. Latruffe N, Delmas D, Jannin B, Cherkaoui Malki M, PassillyDegrace P and Berlot JP: Molecular analysis on the chemopreventive properties of resveratrol, a plant polyphenol microcomponent. Int J Mol Med 10: 755-760, 2002. 
6. Lee JY, Huerta-Yepez S, Vega M, Baritaki S, Spandidos DA and Bonavida B: The NO TRAIL to YES TRAIL in cancer therapy (Review). Int J Oncol 31: 685-691, 2007.

7. Bonavida B, Ng CP, Jazirehi A, Schiller G and Mizutani Y: Selectivity of TRAIL-mediated apoptosis of cancer cells and synergy with drugs: the trail to non-toxic cancer therapeutics (review). Int J Oncol 15: 793-802, 1999.

8. Eid MA, Lewis RW, Abdel-Mageed AB and Kumar MV: Reduced response of prostate cancer cells to TRAIL is modulated by NFkappaB-mediated inhibition of caspases and Bid activation. Int J Oncol 21: 111-117, 2002.

9. Chaudhari BR, Murphy RF and Agrawal DK: Following the TRAIL to apoptosis. Immunol Res 35: 249-262, 2006.

10. Bagnoli M, Canevari S and Mezzanzanica D: Cellular FLICEinhibitory protein (c-FLIP) signalling: a key regulator of receptor-mediated apoptosis in physiologic context and in cancer. Int J Biochem Cell Biol 42: 210-213, 2010.

11. Shylesh BS and Padikkala J: In vitro cytotoxic and antitumor property of Emilia sonchifolia (L.) DC in mice. J Ethnopharmacol 73: 495-500, 2000.

12. Muko KN and Ohiri FC: A preliminary study on the antiinflammatory properties of Emilia sonchifolia leaf extracts. Fitoterapia 71: 65-68, 2000.

13. Shylesh BS and Padikkala J: Antioxidant and anti-inflammatory activity of Emilia sonchifolia. Fitoterapia 70: 275-278, 1999.

14. Tang YJ, Yang JS, Lin CF, et al: Houttuynia cordata Thunb extract induces apoptosis through mitochondrial-dependent pathway in HT-29 human colon adenocarcinoma cells. Oncol Rep 22: 1051-1056, 2009.

15. Chang YH, Yang JS, Kuo SC and Chung JG: Induction of mitotic arrest and apoptosis by a novel synthetic quinolone analogue, CWC-8, via intrinsic and extrinsic apoptotic pathways in human osteogenic sarcoma U-2 OS cells. Anticancer Res 29: 3139-3148, 2009.

16. Chou LC, Yang JS, Huang LJ, et al: The synthesized 2-(2fluorophenyl)-6,7-methylenedioxyquinolin-4-one (CHM-1) promoted G2/M arrest through inhibition of CDK1 and induced apoptosis through the mitochondrial-dependent pathway in CT-26 murine colorectal adenocarcinoma cells. J Gastroenterol 44: 1055-1063, 2009.

17. Lu HF, Lai KC, Hsu SC, et al: Curcumin induces apoptosis through FAS and FADD, in caspase-3-dependent and -independent pathways in the N18 mouse-rat hybrid retina ganglion cells. Oncol Rep 22: 97-104, 2009.
18. Yodkeeree S, Sung B, Limtrakul P and Aggarwal BB: Zerumbone enhances TRAIL-induced apoptosis through the induction of death receptors in human colon cancer cells: Evidence for an essential role of reactive oxygen species. Cancer Res 69: 6581-6589, 2009.

19. Yang JS, Hour MJ, Huang WW, Lin KL, Kuo SC and Chung JG: MJ-29 inhibits tubulin polymerization, induces mitotic arrest and triggers apoptosis via CDK1-mediated Bcl-2 phosphorylation in human leukemia U937 cells. J Pharmacol Exp Ther 334: 477-488, 2010.

20. Lo C, Lai TY, Yang JH, et al: Gallic acid induces apoptosis in A375.S2 human melanoma cells through caspase-dependent and -independent pathways. Int J Oncol 37: 377-385, 2010.

21. Irving BA, Weltman JY, Brock DW, Davis CK, Gaesser GA and Weltman A: NIH ImageJ and Slice-O-Matic computed tomography imaging software to quantify soft tissue. Obesity (Silver Spring) 15: 370-376, 2007.

22. Borner MM: Molecular targets in colon cancer. Ther Umsch 63: 243-248, 2006.

23. Huerta S, Goulet EJ and Livingston EH: Colon cancer and apoptosis. Am J Surg 191: 517-526, 2006.

24. Tao J, Di L, Wen H, Zhao X, Shan J and Cai B: Investigation of research method of fingerprint pharmacodynamics of traditional Chinese medicines. Zhongguo Zhong Yao Za Zhi 34: 2410-2413, 2009.

25. Tan KY, Liu CB, Chen AH, Ding YJ, Jin HY and Seow-Choen F: The role of traditional Chinese medicine in colorectal cancer treatment. Tech Coloproctol 12: 1-6, 2008.

26. Kawahara K, Oyadomari S, Gotoh T, Kohsaka S, Nakayama H and Mori M: Induction of CHOP and apoptosis by nitric oxide in p53-deficient microglial cells. FEBS Lett 506: 135-139, 2001.

27. Chen JJ, Chou CW, Chang YF and Chen CC: Proteasome inhibitors enhance TRAIL-induced apoptosis through the intronic regulation of DR5: involvement of NF-kappa B and reactive oxygen species-mediated p53 activation. J Immunol 180: 8030-8039, 2008.

28. Pennarun B, Meijer A, de Vries EG, Kleibeuker JH, Kruyt F and de Jong S: Playing the DISC: turning on TRAIL death receptor-mediated apoptosis in cancer. Biochim Biophys Acta 1805: 123-140, 2010.

29. Billen LP, Shamas-Din A and Andrews DW: Bid: a Bax-like BH3 protein. Oncogene 27 (Suppl. 1): S93-S104, 2008. 